\title{
Evidence for a new species of Cryptosporidium infecting tortoises: Cryptosporidium ducismarci
}

Donato Traversa

\begin{abstract}
Cryptosporidiosis affects the gastrointestinal and respiratory tract of humans as well as of a wide range of companion, farm, laboratory and wild animals. In the past few years, three independent studies have provided strong evidence for the existence of a distinct Cryptosporidium species affecting tortoises and likely circulating in other reptile species as well. A new Cryptosporidium genotype was firstly detected and genetically characterized in a marginated tortoise in Italy in 2007 and named Cryptosporidium sp. ex Testudo marginata CrIT-20. The phylogenetic analysis of this isolate indicated that this Cryptosporidium was unique and belonged to the intestinal clade. These findings were later on confirmed by the detection of genetic homologies of isolates from a python and a chameleon from Spain and by recent research in the United States. The latter study presented both the occurrence of intestinal lesions in a pancake tortoise and a Russian tortoise and the genetic characterization of the isolates, together with the first pictures of the endogenous stages of Cryptosporidium CrIT-20. Phylogenetic inference based on the sequences representing small subunit of the nuclear ribosomal RNA gene (SSU) of these isolates confirmed the pathological findings because this Cryptosporidium was related to the intestinal group and supported previous results in T. marginata from Italy. The present scientific data on the Cryptosporidium CrIT-20 support its classification as a new species of Cryptosporidium causing intestinal diseases in tortoises. Although further morphological (i.e. exogenous stages) and biological aspects (i.e. complete host range) are yet to be elucidated, it is proposed that this Cryptosporidium is designated Cryptosporidium ducismarci.
\end{abstract}

\section{Findings}

This report aims to propose a new species of Cryptosporidium isolated from reptiles. Cryptosporidium spp. are apicomplexan parasites of a wide range of animals. Due to their biology, ecology and epidemiology these protozoa are globally distributed. The vertebrate hosts become infected through host-to-host contact or through ingestion of contaminated food or water [1,2]. The taxonomy of Cryptosporidium has been debated and several doubts and uncertainties still exist. For a long time the only recognized species have been Cryptosporidium parvum and Cryptosporidium muris. However, numerous other isolates were present in animals but described only in the last decades [3]. The difficulties in addressing Cryptosporidium taxonomy and in delineating new species mainly rely on the inability to morphologically discriminate the biological stages and on the difficulties in establishing monospecific experimental infections [3]. With the advent of nucleic acid-based

\footnotetext{
*Correspondence: dtraversa@unite.it

1 Department of Comparative Biomedical Sciences, University of Teramo, Italy Full list of author information is available at the end of the article
}

techniques and sequencing, important results have been achieved in elucidating the taxonomical status of several Cryptosporidium isolates and presently 19 distinct species are known to affect amphibians, reptiles, birds and mammals $[2,4]$. Three more species are hypothesized to infect fishes but their status is not supported by sound biological and molecular data $[2,4]$. Indeed, the genetic characterization, at one or more loci, of Cryptosporidium is a fundamental requirement to assess, at the species level, a new Cryptosporidium isolate, along with the morphological description of the biological stages and definition of the host range, possibly based upon natural and experimental infections $[2,4]$.

Non-conventional animals, such as reptiles, have increased in popularity as pets in developed countries, posing new concerns in relation to their pathogens. Despite reptile cryptosporidiosis being recognized since the 1970s [5] the first described species (i.e. Cryptosporidium serpentis), which is able to cause significant gastric pathologies in snakes, has been genetically described only recently $[6,7]$. Subsequently, Cryptosporidium varanii/saurophilum, 
whose taxonomic identity has been debated for some time, has been elevated as a proper species of intestinal pathogen of lizards [2,8]. Beyond these two species, other isolates have been reported sporadically and partially described from different reptile species [9-11]. Indeed, our understanding of Cryptosporidium affecting chelonians is very limited. The reports of Cryptosporidium spp. in Testudines published in the last fifteen years relate to the infection in the following species: radiated tortoise (Geochelone radiata), Indian star tortoise (Geochelone elegans), travancorelike tortoise (Indotestudo spp.), gopher tortoise (Gopherus polyphemus), spur-thighed tortoise (Testudo graeca), Egyptian tortoise (Testudo kleinmanni) and Hermann's tortoise (Testudo hermanni) $[9,10,12,13]$, but no comprehensive data on multi-locus genetic characterization, infection site/s or biological aspects were generated. For example, in a study performed in 1997 in the United States, G. radiata, G. elegans, Indotestudo sp. and G. polyphemus were found positive at microscopic and immunofluorescence assays [13] which, however, have important limits in terms of specificity and do not allow a discrimination at the species/ genotype level. Another key example is provided by the fatal intestinal pathology ascribed to Cryptosporidium spp. in T. kleinmanni in 1998, which was not supported by the genetic characterization of the pathogen [12].

In the past few years, three independent studies have provided strong evidence for the existence of a distinct Cryptosporidium species affecting tortoises and probably circulating in other reptile species as well. The first clue for the presence of this species was published in 2008: a genetic isolate of Cryptosporidium has been found and characterized from the faeces of a marginated tortoise (Testudo marginata) living in an open enclosure of central Italy [14]. A multi-locus genetic analysis via the characterization of informative regions within the genes encoding the Cryptosporidium oocyst wall protein (COWP) and the small subunit of the nuclear ribosomal RNA (SSU rRNA) was conducted. The COWP and SSU sequences revealed only $\sim 80 \%$ and $\sim 94 \%$ identity with the corresponding sequences of the ubiquitous C. parvum (GenBank ${ }^{\mathrm{TM}}$ Accession Numbers XM_627569 and AF108864). Molecular and phylogenetic analyses clearly demonstrated that this isolate was an unique uncharacterized genotype, which has been named Cryptosporidium sp. ex Testudo marginata CrIT-20. The phylogenetic inference showed this isolate had a new COWP sequence at the interface of the two major sister groups (i.e. intestinal C. parvum and gastric C. muris). Further analysis of the COWP amino acid sequence for Cryptosporidium CrIT-20 showed partial common residues with the gastric C. muris (79\% nucleotide identity), as well as, with avian Cryptosporidium baileyi (81\% nucleotide identity) affecting the intestinal and respiratory tract of birds. Also, phylogenetic analysis of the SSU rejected the possibility that Cryptosporidium CrIT-20 is the star tortoise gen- otype $[10,14]$ and confirmed that this genotype is monophyletic with the intestinal Cryptosporidium species [14]. More specifically, the phylogenetic trees showed CrIT-20 genotype being at the bottom of the intestinal species, together with a diverse spectrum of reptilian and avian genotypes and of $C$. baileyi [14]. The hypervariable region of the SSU sequence of Cryptosporidium CrIT-20 (corresponding to the nucleotides 633-703 of C. parvum GenBank $^{\mathrm{TM}}$ Accession Number $\underline{\mathrm{AF} 108864}$ ) revealed high genetic differences when compared with the same sequence of other 28 Cryptosporidium species/genotypes, supporting the CrIT-20 unique position [14].

A second distinct survey performed in 2008 showed the presence of Cryptosporidium CrIT-20 in the faeces of a chameleon (Chamaeleo calyptratus) and a python (Python regius) in Spain [11]. The neighbour-joining analysis of partial SSU sequence data confirmed this Cryptosporidium clustering with the species affecting the intestine but representing a distinct clade. Nonetheless, the characterization of a fragment within the gene encoding the $70 \mathrm{kDa}$ heat shock protein indicated this Cryptosporidium to be related to the gastric species, highlighting the need of further more-indepth study to identify the site of infection. Following the first molecular detection of Cryptosporidium CrIT-20 and its recognition as an unique genotype in T. marginata [14], the Spanish researchers claimed that "a new Cryptosporidium species or genotype closely related to the main intestinal Cryptosporidium species was isolated from a chameleon and a python. This one was found to be related to a further new Cryptosporidium sp. recently identified in a tortoise" [11]. Indeed, the molecular comparison of the SSU sequences generated in Italy [14] and Spain [11] showed a single nucleotidic difference (i.e. a thymine insertion) confirming that the Italian and the Spanish isolates belonged to the same Cryptosporidium genotype. In fact, given the nature of the SSU gene, such a mutation is not taxonomically relevant given that different copies of the SSU gene may have minor intraspecific differences in Cryptosporidium spp. [3].

A third and last study provided new evidence for the existence of a novel species of Cryptosporidium in reptiles [15]. A group of researchers from the USA documented two cases of fatal intestinal cryptosporidiosis in a Malacochersus tornieri (pancake tortoise) and in a Testudo horsfieldii (Russian tortoise) and presented both the occurrence of gut lesions and the genetic characterization of the isolate. The necropsy of the two animals showed intestinal lesions consistent with cryptosporidiosis (i.e. mucosal alteration of the lamina propria and its infiltration with lymphocytes, plasmacells and heterophils). This study [15] provided the first pictures of the intestinal stages of this genetic type of Cryptosporidium. Specifically, numerous round Cryptosporidium stages, of 1-2 $\mu \mathrm{m}$ of diameter, were found closely adhering to the brush border along the intestinal mucosa of 
the Russian tortoise and, analogously, various Cryptosporidium stages with a diameter ranging from 1 to $5 \mu \mathrm{m}$ were present within the cytoplasm of intestinal cells and admixed with mucous in the pancake tortoise as well. Given that SSU is the most investigated genetic locus to address taxonomic questions within the Apicomplexa, including Cryptosporidium, this locus was characterized also for these isolates [15]. Interestingly, the SSU region of the Cryptosporidium isolate from the M. tornieri and the T. horsfieldii in the USA [15] showed a $100 \%$ homology to the sequence of Cryptosporidium CrIT-20 from Italy [14]. The phylogenetic inference confirmed the pathological findings (i.e. site of infection and disease), as the US Cryptosporidium was strongly related to the intestinal group of Cryptosporidium spp. and segregated in a monophyletic clade suggesting its key role in the occurrence of the intestinal infection [15].

Hence, the US study [10] provided the first evidence for an association between the infection by the Cryptosporidium firstly named as Cryptosporidium CrIT-20 and intestinal infection and pathology in tortoises [14,15].

In summary, a new Cryptosporidium genotype was firstly detected and genetically characterized in a marginated tortoise in Italy in 2007 and named Cryptosporidium sp. ex Testudo marginata CrIT-20 with sequences from two loci deposited in GenBank ${ }^{\mathrm{TM}}$ with the Accession numbers EF519704.1 and EF547155.1[14]. No morphological nor biological data on this isolate were generated but the phylogenetic analysis clearly suggested that this Cryptosporidium was unique and belonged to the intestinal group [14]. These findings were later on confirmed by the genetic homologies detected in isolates from asymptomatic $C$. calyptratus and $P$. regius from Spain [11], albeit including a single nucleotidic mutation, considered of negligible relevance here, and, more importantly, by the phylogenetic resolution of the Cryptosporidium stages isolated from the intestine of $M$. tornieri and T. horsfieldii tortoises in USA [15]. On the basis of this knowledge such a Cryptosporidium sp. clearly parasitizes different tortoise species, which appear to be the preferential hosts in which it can cause relevant intestinal pathologies. This Cryptosporidium has been detected in lizards and snakes also [11], although its pathogenic role in these other reptiles is unknown and it can be argued that the infection is occasional. Although further studies are necessary and more information on the morphology and biology of this new Cryptosporidium is required, the multi-locus differences found between Cryptosporidium CrIT-20 and of other established Cryptosporidium spp., which is a basic taxonomic requirement [3], confirm the statement that this genotype is a distinct species.

Thus, the present biological and molecular data on the Cryptosporidium CrIT-20 support to propose it as a new species of Cryptosporidium causing intestinal pathologies in tortoises.
It is proposed that this Cryptosporidium is designated Cryptosporidium ducismarci in memory of General Marco Traversa (born Bari, Italy 26 ${ }^{\text {th }}$ February 1946 - died Bari, Italy $4^{\text {th }}$ January 2009 ) who served for almost 40 years in the Italian National Army (Figure 1).

In accordance with section 8.6 of the ICZN's International Code of Zoological Nomenclature, copies of this article are deposited at the following five publicly accessible libraries: Natural History Museum, London, UK; American Museum of Natural History, New York, USA; Museum National d'Histoire Naturelle, Paris, France; Russian Academy of Sciences, Moscow, Russia; Academia Sinica, Taipei, Taiwan.

\section{Abbreviations}

SSU: small subunit of the nuclear ribosomal RNA gene; rRNA: nuclear ribosomal RNA; COWP: Cryptosporidium oocyst wall protein.

\section{Competing interests}

The author declares that he has no competing interests.

\section{Acknowledgements}

The author is grateful to the Editor-in-Chief and to three anonymous Referees for their constructive comments and suggestions to the manuscript. The author thanks Jan Slapeta and Raffaella lorio for the scientific exchange and

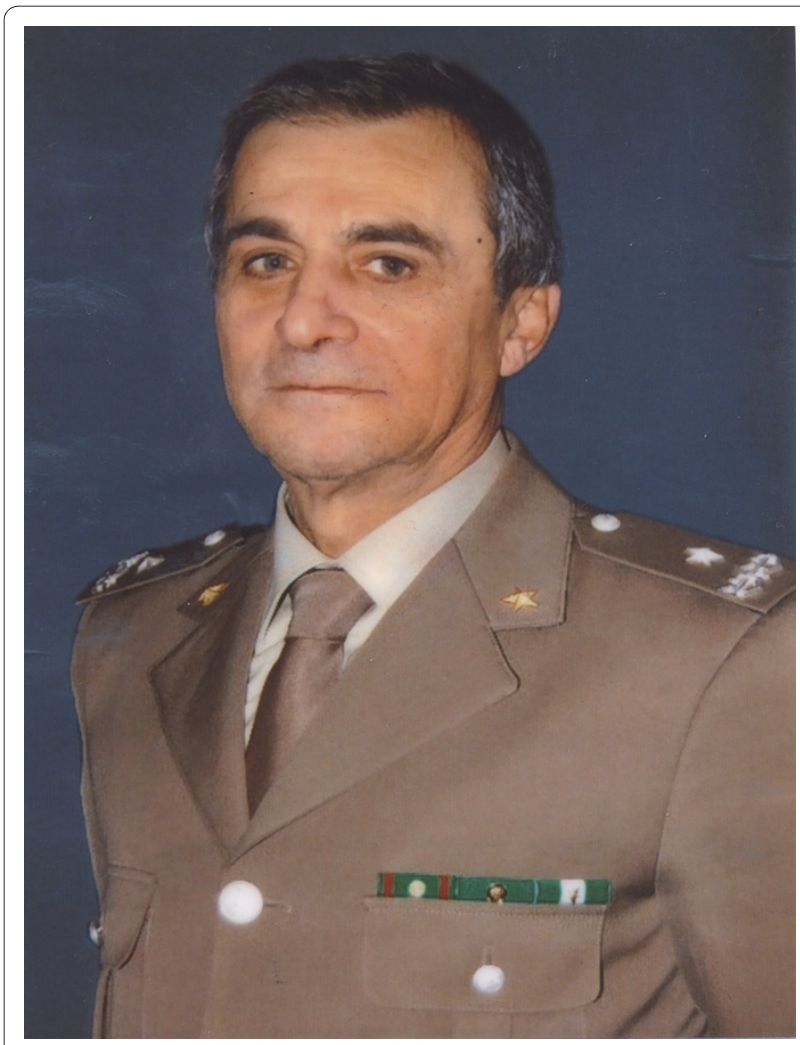

Figure 1 General Marco Traversa. Born Marco Traversa on 26th February 1946, in Bari, Italy. On 19th October 1974 he and Maria Calabrese were married and on $15^{\text {th }}$ October 1975 they welcomed their son Donato. Marco began his career in the Italian National Army on 1968 and retired as a General in 2006. Marco died on $4^{\text {th }}$ January 2009 in Bari, Italy. 

ata CrIT-20.

\section{Author Details}

Department of Comparative Biomedical Sciences, University of Teramo, Italy

Received: 24 February 2010 Accepted: 25 March 2010

Published: 25 March 2010

\section{References}

1. Xiao L, Fayer R: Molecular characterisation of species and genotypes of Cryptosporidium and Giardia and assessment of zoonotic transmission. Int J Parasitol 2008, 38:1239-1255.

2. Fayer R: Taxonomy and species delimitation in Cryptosporidium. Exp Parasitol 2010, 124:90-97.

3. Xiao L, Fayer R, Ryan U, Upton SJ: Cryptosporidium taxonomy: recent advances and implications for public health. Clin Microbiol Rev 2004, 17:72-97.

4. Xiao L: Molecular epidemiology of cryptosporidiosis: an update. Exp Parasitol 2010, 124:80-89.

5. Brownstein DG, Strandberg JD, Montali RJ, Bush M, Fortner J: Cryptosporidium in snakes with hypertrophic gastritis. Vet Pathol 1977, 14:606-617.

6. Xiao L, Escalante L, Yang C, Sulaiman I, Escalante AA, Montali RJ, Fayer R, Lal AA: Phylogenetic analysis of Cryptosporidium parasites based on the small-subunit rRNA gene locus. Appl Environ Microbiol 1999, 65:1578-1583

7. Kimbell LM, Miller DL, Chavez W, Altman N: Molecular analysis of the $18 \mathrm{~S}$ rRNA gene of Cryptosporidium serpentis in a wild-caught corn snake (Elaphe guttata guttata) and a five-species restriction fragment length polymorphism-based assay that can additionally discern C. parvum from C. wrairi. Appl Environ Microbiol 1999, 65:5345-5349.

8. Pavlasek I, Ryan U: Cryptosporidium varanii takes precedence over C. saurophilum. Exp Parasitol 2008, 118:434-437.

9. Xiao L, Ryan UM, Graczyk TK, Limor J, Li L, Kombert M, Junge R, Sulaiman IM, Zhou L, Arrowood MJ, Koudela B, Modrý D, Lal AA: Genetic diversity of Cryptosporidium spp. in captive reptiles. Appl Environ Microbiol 2004, 70:891-899.

10. Alves M, Xiao L, Lemos V, Zhou L, Cama V, da Cunha MB, Matos O, Antunes F: Occurrence and molecular characterization of Cryptosporidium spp. in mammals and reptiles at the Lisbon Zoo. Parasitol Res 2005 97:108-112.

11. Pedraza-Díaz S, Ortega-Mora LM, Carrión BA, Navarro V, Gómez-Bautista M: Molecular characterisation of Cryptosporidium isolates from pet reptiles. Vet Parasitol 2009, 160:204-210.

12. Graczyk TK, Cranfield MR, Mann J, Strandberg JD: Intestinal Cryptosporidium sp. infection in the Egyptian tortoise, Testudo kleinmanni. Int J Parasitol 1998, 28:1885-1888.

13. Raphael BL, Calle PP, Gottdenker N, James S, Linn WJ, McNamara T, Cook RA: Clinical significance of Cryptosporidia in captive and free-ranging chelonians. In Proceedings of the Annual meeting of the American Association of Zoo Veterinarians: 26-30 October 1997; Houston, Texas AAZV 1997:19-20

14. Traversa D, lorio R, Otranto D, Modrý D, Slapeta J: Cryptosporidium from tortoises: Genetic characterisation, phylogeny and zoonotic implications. Mol Cell Probes 2008, 22:122-128.

15. Griffin C, Reavill DR, Stacy BA, Childress AL, Wellehan JFX Jr: Cryptosporidiosis caused by two distinct species in Russian tortoises and a Pancake tortoise. Vet Par 2010 in press.

doi: 10.1186/1756-3305-3-21

Cite this article as: Traversa, Evidence for a new species of Cryptosporidium infecting tortoises: Cryptosporidium ducismarci Parasites \& Vectors 2010, 3:21

\section{Submit your next manuscript to BioMed Central} and take full advantage of:

- Convenient online submission

- Thorough peer review

- No space constraints or color figure charges

- Immediate publication on acceptance

- Inclusion in PubMed, CAS, Scopus and Google Scholar

- Research which is freely available for redistribution

Submit your manuscript at www.biomedcentral.com/submit
C) Biomed Central 